

\section{2 los censos de población en Honduras}

Gladys M enjivar y M arysabel Zelaya

\section{INTRODUCCIÓN}

El C enso es un instrumento y un proceso a la vez que permite obtener datos estadísticos sobre determinados aspectos de la población, se ha realizado desde períodos históricos, en este sentido en H onduras no es la excepción ya que cuenta con una larga tradición censal que se inicia desde el tiempo de la colonia, realizando el primer censo a fines del siglo XVIII (1778) y el último en el año 2001 constituyén dose en uno de los países con mayor número de censos en el contexto latinoamericano.

Este trabajo consiste en revisar la información bibliográfica de los cen sos existentes, conceptualizando el término, tipos de censos, etapas del proceso censal, errores de cobertura y contenido, utilidad de los censos, así como una reseña histórica de los mismos.

A pesar de las dificultades encontradas en el proceso de recopilación de la información se espera que el mismo proporcione información útil sobre el tema abordado y que ayude a los investigadores sobre la sociedad hondureña.

\section{Los censos: historia y proceso}

A I comienzo de la era cristiana la tierra tenía una población estimada entre 133 millones y 300 millones de personas. Pero la poca exactitud en el número de habitantes sugiere el carácter fragmentario, indirecto y poco fidedigno de los datos en que se basan.

A sí como para los primeros años de la civilización esta información era importante, también lo ha sido la época contemporánea. Etimológicamente el vocablo $\mathrm{C}$ enso proviene del latín $\mathrm{C}$ ensus, que significa "padrón o lista que los censores romanos hacían de las persona y haciendas" los primeros gobernantes que real izaron un censo fueron los de las antiguas civilizaciones de Egipto, Babilonia, C hina, Palestina y Roma.

En la Biblia en el capitulo N o. 2, del evangelio de San Lucas Versículo 1 al 5 -, dice lo siguiente: "por aquel tiempo, el emperador C esar A ugusto ordenó que se hiciera un censo de todo el mundo. Este primer censo se hizo sien do Cirenio gobernador de Siria. Todos tenían que ir a empadronarse a su propio pueblo. Por esto. J osé salió del pueblo de $\mathrm{N}$ azaret, de la región de $\mathrm{G}$ alilea, y se fue a Belén, en Judea, donde había nacido el Rey David, por que José era descendiente de David, fue allá a inscribirse, junto con M aría desposada casarse con él, quien se encontraba en cinta" .
Los censos tuvieron un origen y un objetivo diferente al uso que se les da en la actualidad, indica Juan Chakiel:

"Estaban destinados a empadronar a las personas con fines militares o con el fin de empadronar a la población y de organizar la recaudación de impuestos personales. Con este objetivo se sabe de la existencia de formas primarias de estadística y censos desde los inicios de la civilización. A lgunas fuentes ubican el censo más antiguo en China en el año 2238 antes de C risto, ordenado por el emperador Yao para empadronar a la población y las labores de cultivo.

En Roma, el C ensus comenzó a ser efectuado durante la época de la Republica para determinar la composición de las diversas clases y estamentos de la sociedad, cuyos derechos políticos eran muy variables.

En la historia de los censos han existido diferentes formas de cuantificar a la población: maravedis, fuegos, vecinos, casas, almas 0 habitaciones, según las Cortes de Tarazona, de 1495, " un fuego" estaba constituido por " todas aquellas personas que habitan en una casa y toman la despensa de un superior o pater familias continuamente en dicha casa, lo que ha de jurar el superior o pater familias" después el termino "vecino" se utilizó para designar a quienes estaban a la cabeza de las familias.

En Latinoamérica también hay muestra de estos procesos en la historia del Imperio Incaico, que enriqueció su cultura con la información estadística, en los aspectos demográfico y económico, testimonio de esas informaciones es el "quipu", un sistema que permitía asegurar la adecuada relación entre el crecimiento de la población y la distribución de las tierras de cultivo, ya que el crecimiento del número de miembros de la familia determinaba la entrega de una mayor extensión de tierras para la agricultura.

En España durante la época virreinal se realizaron censos sólo con fines tributarios. A través de este sistema los funcionarios de la corona española sabían exactamente cuántas personas debían pagar tributos.

El interés de los poderes públicos por conocer el volumen de la población fue constante durante lo que se conoce como la Edad M oderna, pero continúo motivado más por preocupaciones fiscales 0 militares que por las solamente demográficas. Eso tradujo en la impopularidad que rodeaba a los censos en aquel entonces.

En 1753 la Cámara de los Comunes todavía rechazaba un proyecto de $C$ enso $G$ eneral, entre otras razones, porque amenazaba las 
libertades inglesas. D urante el siglo XV III se comenzó a considerar la población como una variable de conocimiento necesario para planificar la acción política.

Poco a poco se fueron realizan do los primeros cen sos con criterios modernos. Suecia introdujo, a partir de 1749, la periodicidad de Ios recuentos que tardó en imponerse de forma generalizada.

$\mathrm{N}$ o fue hasta el siglo XIX en que, de modo más práctico y exacto, se empezó a hablar de "almas" o "habitantes". Y sólo a partir de 1860 comenzaron a realizase los censos como se les conocen en la actualidad.

\section{Los censos: tipos y características}

Las $\mathrm{N}$ aciones $\mathrm{U}$ nidas definen un censo como el proceso total de recolectar, compilar, evaluar, analizar y publicar datos demográficos en un momento en el tiempo, para todas las personas de un país 0 una región determinada de un país. A unque el censo es una fotografía de la población en un momento del tiempo, también suministra las bases para hacer referencia sobre el pasado y futuro de una población.

Los censos tienen como propósitos los siguientes:

a) Brindar un inventario sobre los recursos y características de un país en un momento dado.

b) Suministrar información a muchas dependencias en el gobierno y sector privado.

Los principales usos que ofrecen los censos son los de realizar proyecciones de población, para estimaciones de datos demográficos y suministrar datos para interrelaciones.

Para proyecciones de Población: Los censos son considerados como el proceso más exhaustivo para registrar los cambios en la magnitud y composición de la población en dos momentos del tiempo. Dichos datos son esenciales para las proyecciones de población.

Para estimaciones de datos demográficos: Los censos también suministran datos que posibilitan el estudio de las interrelaciones entre las características demográficas y socioeconómicas de individuos y hogares.

$\mathrm{H}$ ay dos tipos de censos de acuerdo a la asignación de la población en las distintas áreas territoriales, de jure o derecho y de facto 0 de hecho.

El censo de derecho asigna la población al área donde reside habitualmente, independientemente en donde se haya en el momento del censo. Este tipo de censo refleja con mayor aproximación la verdadera distribución de la población en el territorio. Tiene el inconveniente de que debe definirse con mucha claridad lo que entiende por "residencia habitual".

El censo de hecho atribuye la población al lugar donde se encontraba en el momento de referencia utilizado en el empadronamiento. Según sea el criterio adoptado se presentan diferencias en las cifras relativas a la distribución geográfica de la población, lo que afecta los estudios de migración interna y la constitución de los hogares y las familias. La tendencia en A mérica Latina es efectuar cen- sos de "facto" o de hecho. En los países donde se practican los cen sos de hecho se debe tomar precaución de hacerlo en un momento en que haya poca movilidad de la población.

A I momento de referencia utilizado en el empadronamiento se le denomina "momento censal". A unque el censo tarde una semana o más para ser finalizado, toda la información recogida se referirá a un instante, Ilamado "momento censal", que general mente es la cero hora del día en que se inicia el empadronamiento.

Este requisito se conoce como de simultaneidad. Por consiguiente que este es un aspecto estático de la población. Q ue puede comparase con una fotografía de la población.

Las principales características U niversales de un censo de población son seis y son las siguientes: U niversalidad, simultaneidad, secreto estadístico, individualidad o unidad censal, territorio definido y auspicio del estado.

Es imprescindible que toda la población del territorio censal definido sea empadronada, nadie debe de ser exclusivo.

Es necesario que la información esté referida a un momento preciso del tiempo.

Los datos que se recogen no pueden servir para identificar personas especificas bajo ningún pretexto. Si en la boleta censal se pregunta el nombre de los empadronados es como mecanismo de "familiarización", entre el entrevistado y el empadronador y también para facilitar el listado y la contabilización de los empadronados.

En un censo de población la unidad censal esel individuo, y en el de vivienda, es la vivienda. Esto no implica, la imposibilidad de efectuar preguntas sobre el hogar como un todo. De hecho, la unidad de referencia de las boletas censales, es normalmente, el hogar particular.

Debe ejecutarse el censo en un territorio o zona claramente establecida. Un censo nacional de población puede excluir ciertas regiones del país por razones de seguridad o accesibilidad, pero debe establecer precisamente los limites de las áreas que serán cen sadas y las que no serán.

Los censos son patrocinados por el Estado, independientemente que la agencia ejecutora sea gubernamental o privada.

A ctualmente existen tres recomendaciones internacionales para el levantamiento de censos:

- Q Que se levanten los censos simultáneamente a todos los países y en años terminados en cero.

- Que tenga una periodicidad constante, cada diez años.

- Que se ejecuten conjuntamente el censo de población y el de vivienda.

La planificación total de un censo consta de tres etapas: la de preempadronamiento, la de empadronamiento y la de post empadronamiento:

a) El pre-empadronamiento incluye una frase previa en la cual, el censo es sancionado legalmente por decreto. Se estructura la operación en términos administrativos y se define un cronograma ini- 
cial de actividades. También se realiza un trabajo geográfico o cartográfico que actualiza los mapas nacionales y determina las zonas censal es utilizando criterios relacionados con el clima, topografía, transporte, etc. Conjuntamente se definen los temas y preguntas censales. Q ue deben de ser pocas y lo más precisas posibles.

Se contempla en esta etapa, los ensayos del cuestionario, las pruebas de campo y censos pilotos en zonas seleccionadas. Ello permite eval uar los requerimientos concretos que existirán al momento de la operación definitiva, calcular el tiempo medio de respuestas y medir la calidad de las preguntas.

También se incluye en esta etapa la preparación del personal que levantará la información mediante capacitaciones sobre aspectos metodológicos y conceptuales básicos. Luego el levantamiento del censo viene la etapa de post empadronamiento, que incluye la recepción y verificación de los cuestionarios, la codificación (manual por lectura óptica, la aplicación de programas de criticas y asi g- nación, el procedimiento estadístico (obtención de tabulados) y la publicación de tabulados. A veces se considera la realización de una encuesta de evaluación, con el fin de verificar errores de cobertura y calidad de la información. En algunas ocasiones se ha utilizado el muestreo para adelantar resultados provisionales. Los resultados de un censo se van hacien do públicos de manera escalonada.

b) El empadronamiento: se realiza en forma simultanea en todo el país, este es el momento en el cual se recoge la información censal.

c) Fase post empadronamiento: comprende la captura de los datos para el procesamiento de la información censal utilizando algunos programas especiales

Sin embargo existen una serie de ventajas y limitaciones respecto a la aplicación y utilización de los censos, sobresalen las siguientes:

\section{Ventajas}

1) Los datos pueden tabularse para muchas áreas geográficas, regionales y locales.

2) Las tabulaciones detalladas no están sujetas a errores de muestreo para enumeraciones completas y a errores relativamente bajos para variables por muestreo.

3) Obtiene información de forma simultanea para los eventos y la población en riesgo.

4) Útil para análisis dinámico (en el tiempo) para períodos largos.

\section{Limitaciones}

1) Poco frecuente.

2) Cobertura y profundidad limitada en la recolección de datos sobre fecundidad, mortalidad y en las variables de clasificación.

3) La información sobre variables tales como ingreso, nacimiento, defunciones y la obtenida de personas diferentes al sujeto presentan niveles altos de no respuesta.

4) Las personas fuera de su lugar de residencia habitual están expuestas a no ser enumeradas. 


\section{Censos Realizados en Honduras entre $\mathbf{1 7 7 8}$ y el año $\mathbf{2 0 0 0}$}

Como se ha dicho en el inicio de este trabajo $\mathrm{H}$ onduras es un país que cuenta con acumulado de información muy importante en los cen sos. Prácticamente desde la segunda mitad del siglo todas las décadas se realizó un censo. A continuación se presentan las características e información existente en cada uno de estos censos.

$1778 \rightarrow$ El primer $\mathrm{C}$ enso realizado en $\mathrm{H}$ onduras fue el de los indígenas tributarios en ocasión de la introducción de las Leyes de Indias; además, la C apitanía $\mathrm{G}$ eneral de $\mathrm{G}$ uatemala hizo un censo real del país, que dio como total de habitantes la cantidad de 88,143 mil habitantes distribuidos entre Comayagua, Tegucigal pa y $C$ astillo de 0 moa. Este $C$ enso consistió en una revisión sistemática de los registros parroquiales. No se incluyó a la población de la M osquitia $\mathrm{ni}$ a las regiones montañosas.

$1791 \rightarrow$ Este censo fue levantado por el obispo Fray Fernando de C ardiñatos, en dos visitas realizadas en los años de 1789 y 1791; el total de la población era de 96,421 habitantes distribuidos en 35 curatos o parroquias. La diócesis se componía de 135 poblaciones y 231 valles.

$1801 \rightarrow$ El C enso de este año fue ordenado por el G obernador Intendente y Comandante $\mathrm{G}$ eneral de la Provincia de Honduras, don Juan Ramón A nguiano, del cual resultó que la población del país era de 128,453 habitantes. Dicha información se obtuvo mediante la recopilación de datos sobre el número de familias de españoles, ladinos, indios, solteros que habían en cada pueblo o parroquia que debían tributo. Y la mayor parte de la población que se detalla se encuentra en el partido de Olancho, indios y zambos, que son irreconciliables enemigos y su número asciende a 60,000 aunque no se tenía la certeza; en el mismo partido se encuentran situados los indios payas en número de diez a doce mil y en el partido de Yoro los indiosjicaques en número de diez y seis mil.

Enero de $1821 \rightarrow$ Este Censo consistió en un resumen cartográfico e histórico de $\mathrm{G}$ racias levantado por don José M aría Cacho y el censo de población de la Villa de Tegucigalpa, el cual enumeró características tales como:

- N ombre, Edad, 0 ficios.

- $\quad$ El total de 488 casas.

- En este censo se averiguó de modo exacto el número de pueblos y personas que deberían pagar tributos.

Los censos demográficos modernos comienzan a partir de 1880 bajo la influencia del liberalismo, el cual crea el departamento de Estadística $\mathrm{N}$ acional.

$1881 \rightarrow$ El doctor Francisco C ruzfundador y primer director de estadística levantó el primer censo de población en 1881, publicado el 31 de diciembre de 1882 mediante la justificación de que " por ser el censo la primer base de toda estadística, con preferencia me ocupé de formular los modelos de los cuales y de resumir suficiente cantidad de ejemplares impresos con amplias y claras instrucciones a las oficinas sucursales para el empadronamiento metódico y uniforme en todos los pueblos de la república en términos de poder conocer con la exactitud posible la población en general". El primer censo nacional contó con 307,289 mil habitantes, con un porcentaje muy alto de sub-enumerados debido a la oposición de los indígenas a ser contados. Tal censo incluyó la siguiente información:

- $\quad$ Número de casas y edificios según departamento.

- Población por grupos de edad según sexo y departamento.

- Población clasificada según profesionales y oficio.

- Población según educación (sobre leer, sobre escribir).

- Estado civil (soltero, casado, viuda).

- Capacidad política (ciudadanos electores, conciudadanos, elegibles y empleados públicos).

- Inmigración (migrantes, no migrantes).

Junio $1887 \rightarrow$ el primero de junio de 1887 se levantó el censo de población (se investigó también el numero de habitantes). Publicado el 29 de octubre de 1888 siendo el Director G eneral de Estadística el Dr. A ntonio R. Vallejo. Dicho censo sumó 331,917 mil habitantes con el dato corregido a 381,938 mil habitantes; enumeró Ias siguientes características: pueblo, ciudad villa, aldea, caserío, área urbana o rural.

Á rea urbana 22 ciudades, 19 villas, 179 pueblos, 679 aldeas, y 327 caseríos considerados como área rural. Se presentó a nivel de municipio, por área, la población según grupo de edad, estado civil; se detalla además: nacionalidad étnica, religión, instrucción, defecto físico, capacidad política, profesión, arte e industria.

Este censo tiene error de cobertura, ya que presento una omisión de 50,000 personas.

$1895 \rightarrow$ Este enumeró un total de 398,877 habitantes. Los datos se presentaron tabulados conteniendo información de hondureños, extranjeros y población total por departamento. Este censo fue levantado en diciembre siendo Director General de Estadística el señor Eduardo Guillén.

$1901 \rightarrow$ Tiene error de omisión de 10\%. Se publicó en agosto de 1902, siendo Director de Estadística y Censo el señor Teofilo $C$ anales. A quí se incluyeron datos sobre el número de milicianos y proletarios obligados a prestar su servicio personal en los caminos.

$1905 \rightarrow$ Se realizó el 31 de diciembre y se publicó en abril de 1906 siendo el Director G eneral de Estadística el señor M aximiliano Sagastume. Se incluyó información total por sexo y número de casas según departamento y municipio.

$1910 \rightarrow$ Este censo fue levantado el 18 de diciembre de 1910 y publicado en 1911, siendo Director General de Estadística el señor Teofilo $C$ anales. En él se hizo un recuento del número de casas y habitantes por departamento.

- Presentó una sub-enumeración de 6 a 10\%.

$1916 \rightarrow$ Se levantó el 17 de diciembre de 1916 siendo Director $G$ eneral de Estadística el señor A . N avarro. Dicho censo obtuvo información acerca de los habitantes, casas particulares y edificios públicos que había ese momento. 
- se considera una sub-enumeración de 6 a 10\%.

$1926 \rightarrow$ Levantado el 26 de diciembre de 1926 y publicado el 31 de mayo de 1927, siendo Director de Estadística el señor H éctor Pineda; aquí se registró en forma sistemática y se comienza a registrar el lugar de nacimiento de los habitantes; se publicó como datos la nacionalidad, los domiciliados y transeúntes por sexo, municipio y departamento.

- Tiene una sub-enumeración de 6 a $10 \%$ y fue corregida (por el CELADE) al $26.6 \%$.

$1930 \rightarrow$ Se realizó el 29 de junio de 1930 y se publicó el 31 dejulio de 1931 siendo Director General de Estadística el señor José Pineda; este censo fue considerado el mejor hasta entonces, investigó además de los datos de población el número de casas; los tabulados se presentaron según los tipos de áreas, ya sea urbana 0 rural y aparecen las categorías de divorciado - unión libre.

- Retomó la investigación de raza, nacimiento, estado civil, religión, ocupación, bienes raíces e información sobre la asistencia escolar de la población de 7 a 15 años.

- $\quad$ Este censo fue de facto.

- Se considera el más correctamente enumerado.

- Tiene una sub-enumeración del $11 \%$.

$1935 \rightarrow$ Este censo se levantó el 30 de junio de 1935 y se publicó el 2 de mayo de 1936 siendo Director General de Estadística Enrique B. U clés; contiene la población por edad, estado civil, raza, nacionalidad, profesiones u oficios, religión, defectos físicos y mentales, bienes raíces y número de familias. Tal censo tuvo un formato semejante al de 1930.

- No se especificó la información de área urbana - rural y se considera una sub-numeración de 3 a 6\%. Se corrigió a $6.6 \%$. $1940 \rightarrow$ Serealizó el censo el 30 de junio de 1940 siendo publicado en el informe de G obernación, Justicia, Sanidad y Beneficencia de 1940 -1941. En aquel entonces era el Director General de Estadística y $C$ enso el señor M iguel A ngel C ruz. Selevantó datos de población por sexo según departamento y municipio.

- Se considera una sub numeración de 3 a 6\%.

$1945 \rightarrow$ Fue levantado el 24 de junio de 1945 y publicado en 1947 siendo Director General de Estadística don Vicente Palma. En este censo por primera vez se preguntó por la escolaridad primaria.

- $\quad$ Se publicaron datos sobre la condición legal, sexo, edad, estado civil, raza, nacionalidad, profesión, oficios, al fabetismo, asistencia escolar, religión, defectos físicos y mentales, y datos sobre propietarios de bienes raíces inmuebles.

- Se considera una sub-enumeración de 3 a $6 \%$.

1949 - $1950 \rightarrow$ Se realiza el primer censo de población y vivienda, en esta ocasión los datos se levantaron separados.

- $\quad$ El de vivienda es de fecha 1 de julio de 1949 y publicado el 21 de diciembre del mismo año siendo Director General de Estadística el Profesor G ustavo Castañeda; se publicaron datos sobre la clase uso, condición y ocupación de la vivienda, mate- rial de losmuros y paredes, material del piso, techo, provisión de agua, servicios sanitarios, iluminación y combustible usado.

- El 10 de Junio de 1950 se levantó el censo de población, siendo Director de Estadística el señor Carlos Zúñiga Figueroa.

- Estos fueron los momentos censales, posteriormente se unieron los dos aspectos.

- Se publicó la población por edad, estado civil, nacionalidad, educación, y la cantidad de miembros de la familia censal. A demás la PEA de acuerdo a lo siguiente:

a. por rama de actividad económica.

b. por clase de ocupación.

c. categoría ocupacional.

d. población que usa y no usa calzado.

e. población que come y no come pan.

f. y según clase de adminículo en que duerme.

- $\quad$ Se produjeron tabulaciones según departamento de nacimiento; aparece la categoría de casado - separado.

- Se considera una sub-enumeración de 3 a $6 \%$.

$1961 \rightarrow$ Este se levantó en abril de 1961 y se publicó en diciembre de 1964 siendo D irector $G$ eneral de Estadística y C ensos el señor JoséTrinidad Fiallos. En este censo se consideraron como zona urbana los centros poblados con 1000 habitantes o más y que tuvieran los siguientes servicios:

a. Escuela primaria completa

b. Por lo menos uno de los servicios siguientes:

Correo, telégrafo o teléfono público,

c. Comunicación terrestre (carretera o ferrocarril) o servicio regular aéreo o marítimo,

d. Servicio de agua de cañería

e. Servicio de alumbrado eléctrico

Este censo se publicó en dos volúmenes. El primero se refiere a las características generales y educativas de la población y el segundo a las características económicas.

Fue un censo de facto y se tuvieron en cuenta las recomendaciones indicadas en el programa del censo de 1960. Se considera el más correctamente en umerado:

- Se considera una sub enumeración de $9.5 \%$ en cuanto a vivienda y de $8.9 \%$ en cuanto a personas.

- Se optó por una corrección de 5.3\% a 5.6\%.

$1974 \rightarrow$ Este censo depoblación y vivienda fue levantado el 6 de marzo de 1974 y publicado en 1976 siendo Director de Estadística y Censos la licenciada Leticia Ma Tay; el censo fue de jure o de derecho y se tomaron en cuenta los principios y recomendaciones de la catorceava sesión de la comisión de estadística de las $\mathrm{N}$ aciones U nidas de 1966, así como las del Consejo Nacional de Estadística.

En esta oportunidad se consideraron urbanos los centros poblados con 2,000 y más habitantes y que tuvieran los cuatro siguientes servicios: agua de cañería, vías de acceso, escuela primaria completa y servicios de correo o telecomunicaciones y por lo menos uno 
de los siguientes servicios: Iuz eléctrica, alcantarillado o centro de salud.

- Se incorporó la pregunta sobre el lugar de residencia,(de cinco años por lo menos) antes de la enumeración.

- No aparece información sobre el lugar de nacimiento de las personas según el departamento de residencia al momento censal, ni tampoco año de llegada al país entre las características de la población extranjera.

- Se midió la profundidad y la mortalidad infantil (orfandad materna).

- Limitantes:

- No había dato sobre migración.

- $\quad$ No permite conocer las poblaciones por departamento clasificadas simultáneamente por edad simple y sexo.

- La información departamental sobre la estructura por edad sencilla no se tabuló separadamente para los censos. Lo que impidió el cálculo por separado de losíndices de los hombres y mujeres. $1988 \rightarrow$ Este censo fue jure o de derecho.

- Se incluyó los temas del censo de 1974, incorporando nuevas preguntas y nuevos temas tales como:

- Identificación de productores agropecuarios para obtener un marco de referencia para el censo agropecuario.

- Se incorporó la pregunta para conocer habitantes de lenguas in dígenas y garífunas. Los factores de corrección estimadosfueron de $4.57 \%$ para el país como un todo.

- Se considera uno de los más precisos, aunque para G racias a Dios se le calificó de aproximada.

- $\quad$ El momento censal fue a las 12:00 de la noche del día sábado 28 de M ayo de 1988.

- Se preguntó sobre la ubicación geográfica, datos de vivienda, composición de la familia que vive en la vivienda, mortalidad, características de la población.
$2001 \rightarrow$ este censo fue de Jure o de Derecho. El momento censal fue el 28 dejulio de 2001, siendo directora la Licenciada M agdalena García y aún los datos no se han publicado.

Los aspectos que se incluyeron en el censo de población y vivienda fueron:

- U bicación geográfica.

- Datos de vivienda.

- Datos de hogar.

- Composición del hogar.

- Migración internacional y mortalidad.

- Características de las personas.

Consideraciones finales

La historia de los procesos censales se remonta a la edad antigua, su propósito fue militar y tributario, siendo los egipcios babilonios, chinos, palestinos y romanos los primeros en realizarlo.

Los primeros censos realizados en A mérica Latina estuvieron a cargo de la igl esia católica a través de los registros parroquiales.

Los censos demográficos modernos en $\mathrm{H}$ onduras se inician a partir de 1880 con el auge de la reforma liberal es aquí donde se crea el departamento de Censos y Estadística.

A partir de la década de los 30's hasta los 50's se inició una secuencia de censos en nuestro país los cuales se realizaron cada 5 años.

El propósitos de los censos es obtener información actualizada y desagregada necesaria para evaluar los progresos obtenidos, elaborar estrategias o políticas encaminadas a combatir la pobreza y orientar acciones para el desarrollo humano

$\mathrm{H}$ onduras ha tenido una larga tradición censal (18 censos) sin embargo la información proporcionada ha sido subutilizada pues no se le ha dado el uso adecuado en la elaboración de programas y proyectos que impulsen el desarrollo del país. 